\title{
Recommandations pour une reprise équitable après la pandémie de COVID-19 au Canada
}

\author{
Nav Persaud MD MSc, Hannah Woods MSc, Aine Workentin BSc, Itunu Adekoya MSc, James R. Dunn PhD, \\ Stephen W. Hwang MD MSP, Jonathon Maguire MD MSc, Andrew D. Pinto MD MSP, Patricia O'Campo PhD, \\ Sean B. Rourke PhD, Daniel Werb MSc PhD; pour le groupe de travail du Centre MAP pour une reprise \\ équitable après la pandémie
}

Citation : CMAJ 2021 December 13;193:E1878-88. doi : 10.1503/cmaj.210904-f

Voir la version anglaise de la ligne directrice ici : www.cmaj.ca/lookup/doi/10.1503/cmaj.210904; voir l'article connexe ici : www.cmaj. ca/lookup/doi/10.1503/cmaj.211987-f

$\mathbf{L}$ es iniquités en matière de santé, ou les différences évitables de l'état de santé de populations diverses, qui ont été révélées et exacerbées durant la pandémie de COVID-19 pourraient être corrigées par des interventions et des modifications de politiques qui ont fait l'objet d'études avant la propagation du SRAS-CoV-2 partout au Canada ${ }^{1,2}$. Les personnes racisées, les femmes, les personnes à faible revenu, les personnes vivant en situation d'itinérance ou éprouvant des problèmes de toxicomanie et les détenus ont tous été affectés de manière disproportionnée par la pandémi ${ }^{3-7}$. Cette dernière a mis en lumière notre interdépendance à mesure que la propagation du virus parmi les personnes défavorisées menaçait la santé de tous, y compris des privilégiés, et cela a suscité des appels à la lutte aux iniquités sous la forme d'aphorismes tels qu'« aucun d'entre nous n'est en sécurité tant que nous ne le sommes pas tous $»^{1}$.

L'attention portée actuellement aux iniquités qui perdurent depuis longtemps au Canada pourrait s'estomper à mesure que la vaccination contre le SRAS-CoV-2 se déploie et que les risques immédiats de la COVID-19 diminuent. Une action concertée face à la pandémie inclut des efforts pour un retour à la vie « normale » après la menace immédiate ${ }^{8}$, mais la pandémie de COVID19 a rappelé la nécessité de lutter contre les iniquités plutôt que de revenir au statu quo et à ses iniquités. Des mesures de soutien au revenu, un moratoire sur les évictions et d'autres mesures visant à atténuer les effets néfastes de la pandémie ont été temporairement mis en œuvre ${ }^{10}$. Nous y voyons une occasion de recommander des interventions avérées efficaces pour promouvoir l'équité en matière de santé dans le cadre de la reprise postpandémique à plus long terme.

\section{Portée}

Le but de cette ligne directrice est de corriger les iniquités qui ont été exacerbées par la pandémie et de fournir des orientations pour une reprise postpandémique axée sur l'équité en

\section{Points clés}

- Les iniquités qui ont été révélées et exacerbées par la COVID-19 continueront de menacer la santé après la pandémie.

- On sait que des interventions et des modifications spécifiques axées sur le revenu, le logement, la prévention de la violence conjugale, les services de garde, l'accès aux soins de santé et la lutte au racisme ont des effets positifs.

- La mise en œuvre d'interventions et de modifications éprouvées favorisera l'équité en matière de santé et protégera la santé en général pendant la reprise postpandémique et avant la prochaine pandémie.

- Les effets de ces interventions et modifications sur l'équité en matière de santé devront faire l'objet d'un suivi étroit pour éclairer les futures orientations au Canada et ailleurs.

matière de santé en formulant des recommandations touchant le revenu, le logement, la violence conjugale, l'enfance, l'accès aux soins de santé et le racisme. Les populations prioritaires sont celles qui font face à des iniquités précises, indépendamment de leur lieu de résidence au Canada (urbain, rural ou éloigné), et incluent les Néo-Canadiens et les migrants. Les recommandations s'adressent aux décideurs aux échelons fédéral, provincial et municipal chargés d'établir les politiques liées aux iniquités abordées ici et aux professionnels de la santé qui soignent des personnes défavorisées, surtout en médecine de premier recours. L'auditoire cible pourra utiliser ces recommandations pour orienter des politiques ou la prise de décisions cliniques.

La " période de reprise postpandémique » fait référence au moment à partir duquel les effets néfastes directs de la COVID-19 s'estompent; les interventions et modifications recommandées pourraient être nécessaires pendant des mois, des années, voire définitivement, et certaines d'entre elles pourraient être instaurées dès maintenant. 
Les recommandations donnent une indication de l'efficacité de multiples mesures : aide au revenu pour les personnes sans emploi ou à revenu faible, interventions axées sur le logement pour les personnes vivant en situation d'itinérance ou d'instabilité domiciliaire, interventions pour les personnes victimes de violence conjugale, interventions axées sur l'équité en matière de santé infantile, interventions visant à améliorer l'accès aux soins de santé et façons de lutter contre le racisme dans la prestation des soins de santé.

\section{Recommandations}

Nous formulons 13 recommandations fondées sur les conclusions de revues systématiques dans le but de corriger les iniquités en matière de santé en lien avec le revenu, le logement, la violence conjugale, l'enfance, l'accès aux soins de santé et le racisme. La classification des recommandations est expliquée dans l'encadré 1 . L'encadré 2 et la figure 1 résume les recommandations pour la période de reprise postpandémique.

\section{Revenu}

Nous recommandons fortement les transferts de fonds pour assurer un revenu de subsistance qui permette aux gens de combler leurs besoins essentiels comme la nourriture (certitude modérée des effets estimés).

Les transferts de fonds améliorent certains paramètres de santé, comme la probabilité d'avoir souffert d'une quelconque maladie selon une revue systématique de 21 études, dont 16 essais, 4 études d'observation et 1 étude de cohorte, effectués dans des pays à revenu faible et moyen (rapport des cotes [RC] 0,73, intervalle de confiance [IC] à $95 \% 0,57$ à 0,93$)$. (Annexe 1, section $1 \mathrm{~A}$, accessible en anglais au www.cmaj.ca/ lookup/doi/10.1503/cmaj.210904/tab-related-content) ${ }^{12}$. Une récente évaluation des effets du revenu de subsistance en Finlande a fait état d'améliorations de la santé mentale et de la sécurité financière ${ }^{13}$. Certains ont dit craindre que les prestations de revenu de subsistance ne réduisent la motivation à travailler, mais c'est le contraire qui a été observé en Iran et en Finlande ${ }^{13,14}$. Quelques études plus anciennes, comme l'étude de Mincome (Manitoba Basic Annual Income Experiment) des

\section{Encadré 1 : Classification des recommandations ${ }^{11}$}

- «Nous recommandons fortement » signifie que les avantages d'une intervention ou d'une modification surclassent nettement tout effet négatif. La mise en œuvre élargie d'une recommandation pourrait être justifiée dès à présent.

- «Nous recommandons » signifie que les bienfaits d'une intervention ou d'une modification surclassent tout effet négatif. Une mise en œuvre initiale pourrait être évaluée, car les avantages nets peuvent varier.

- Les énoncés entre parenthèses quant à la certitude des effets estimés (certitude élevée, modérée, faible ou très faible des effets estimés) à la fin des recommandations font référence à notre évaluation de la capacité des conclusions des études incluses à illustrer les effets véritables de l'intervention ou de la modification.
Encadré 2 : Sommaire des recommandations pour la période de reprise postpandémique

\section{Revenu}

- Nous recommandons fortement les transferts de fonds pour assurer un revenu de subsistance qui permette aux gens de combler leurs besoins essentiels comme la nourriture (certitude modérée des effets estimés).

- Nous recommandons fortement le versement de prestations universelles d'assurance emploi, de congé parental et de congé de maladie (faible certitude des effets estimés).

- Nous recommandons des formules de crédit ou de prêts abordables pour les personnes à revenu faible (recommandation; très faible certitude des effets estimés).

\section{Logement}

- Nous recommandons fortement l'élargissement des programmes de logements supervisés permanents étroitement liés à l'approche Logement d'abord, ayant les caractéristiques suivantes : engagement volontaire et gestion de cas, moins de $30 \%$ du revenu consacré au logement, choix du logement et des services de soutien, sans obligation de participation des bénéficiaires atteints de problèmes graves de santé mentale, éprouvant des problèmes de toxicomanie ou vivant en situation d'itinérance (forte certitude des effets estimés).

- Nous recommandons d'élargir l'accès aux interventions de prévention des évictions, y compris l'accès à des services juridiques et à des conseils financiers (très faible certitude des effets estimés).

\section{Violence conjugale}

- Nous recommandons fortement des interventions qui incluent l'accès à l'aide juridique et des mesures de soutien aux victimes de violence conjugale (certitude modérée des effets estimés).

\section{Enfance}

- Nous recommandons d'élargir l'accès à des services publics de garderie (faible certitude des effets estimés).

- Nous recommandons la distribution de nourriture saine aux enfants (certitude modérée des effets estimés).

\section{Accès aux soins de santé}

- Nous recommandons d'élargir l'accès au traitement de substitution aujourd'hui appelé « traitement par agoniste opioïde » (TAO) et aux sites d'injection supervisée (certitude modérée des effets estimés).

- Nous recommandons d'élargir le dépistage du virus de l'immunodéficience humaine $(\mathrm{VIH})$ et du virus de l'hépatite $C$ (VHC) aux personnes à haut risque (certitude modérée des effets estimés).

- Nous recommandons l'amélioration des soins de santé pour les personnes incarcérées (faible certitude des effets estimés).

- Nous recommandons fortement d'intégrer les médicaments d'ordonnance dans le système public de santé au Canada (certitude modérée des effets estimés).

\section{Racisme}

- Nous recommandons fortement une réflexion et des actions pour donner suite à de multiples rapports précédents qui proposent des approches pour lutter contre la discrimination à l'endroit des Autochtones, des personnes noires ou d'autres formes de racisme (énoncé non classé). 


\section{Reprise équitable après la pandémie de COVID-19 au Canada}

\section{Problème :}

Les iniquités qui se sont aggravées durant la pandémie continueront de menacer la santé
Solution :

Des changements de politiques aux niveaux fédéral, provincial et municipal pour promouvoir l'équité en matière de santé

\section{Recommandations}

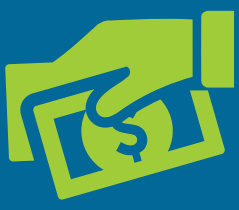

Revenu

- Garantir un revenu de subsistance

- Verser des prestations universelles d'assurance emploi, de congé parental et de congé de maladie

- Donner accès à des prêts abordables

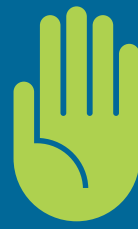
qui incluent l'accès à l'aide juridique et des mesures de soutien aux victimes

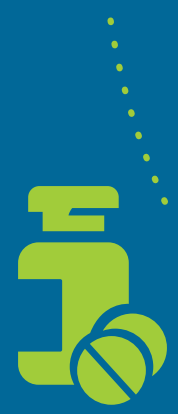

\section{Accès aux soins de santé}

- Élargir l'accès au traitement par agoniste opioïde et aux sites d'injection supervisée

- Élargir le dépistage du VIH et du VHC chez les personnes à haut risque

- Inclure les médicaments d'ordonnance dans le système public de santé au Canada

- Améliorer les soins de santé pour les personnes incarcérées

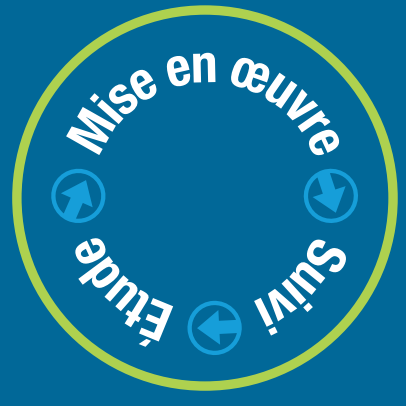

\section{Racisme}

Enfance

- Élargir l'accès aux services publics de garderie

- Distribuer de la nourriture saine aux enfants

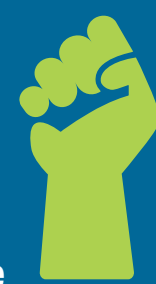

- Mettre en œuvre les recommandations existantes pour vaincre la discrimination envers les Autochtones et le racisme envers les personnes noires 
années 1970, ont montré les avantages du revenu de subsistance, qui incluent une réduction des hospitalisations et une augmentation des taux de diplomation au secondaire ${ }^{15}$. Une récente étude d'observation sur des transferts de fonds mensuels de 500 \$ pendant 2 ans à des personnes à faible revenu à Stockton, en Californie, a montré une augmentation du taux d'emploi, une réduction du poids des dettes et une amélioration de plusieurs paramètres de santé mentale (https://socialprotection.org/ discover/publications/preliminary-analysis-seed's-first-year ${ }^{16}$. Un petit essai récent sur un unique transfert de fonds à des personnes vivant en situation d'itinérance à Vancouver, en ColombieBritannique, a révélé une réduction du nombre de jours d'itinérance ${ }^{17}$. Les transferts de fonds seraient probablement acceptés des bénéficiaires et du public, selon un sondage d'opinion ${ }^{18}$.

Un projet pilote ontarien sur un revenu de base a débuté en 2018 afin de mesurer les effets d'un revenu de subsistance suffisant pour combler les besoins de base comme la nourriture ${ }^{19}$. Le coût d'un tel programme dépendra de l'ampleur du transfert. Des indices comme les paramètres de référence du marché évaluent les besoins essentiels à environ 2000 \$ par mois par adulte, mais le montant varie selon la ruralité et la province ou le territoire ${ }^{20}$. Une approche potentielle au Canada pourrait reposer sur une prestation fédérale de revenu minimum complétée par les provinces et les territoires. Le Bureau du directeur parlementaire du budget a estimé qu'en garantissant un revenu de 16989 \$ par adulte on réduirait les taux de pauvreté de $49 \%$ à un coût d'environ 85 milliards de dollars par année en $2022^{21}$.

Nous recommandons fortement le versement de prestations universelles d'assurance emploi, de congé parental et de congé de maladie (faible certitude des effets estimés).

Toute forme de soutien qui assure un revenu aux travailleurs qui perdent leur emploi améliore la santé autodéclarée, la santé mentale et le bien-être selon une revue systématique de 12 études d'observation ${ }^{22}$ (p. ex., risque de piètre état de santé autodéclaré 3,04, IC à $95 \%$ 2,45 à 3,77 chez les personnes sans emploi qui reçoivent des prestations de base c. 1,25; IC à $95 \%$ 0,89 à 1,75 pour les personnes qui bénéficient de prestations d'assurance emploi; le groupe de référence est formé d'individus actifs sur le marché du travail dans les deux $\operatorname{cas}^{23}$ ) (Annexe 1, section 1B). La perte d'un emploi est associée à un piètre état de santé et l'assurance emploi semble atténuer les effets néfastes du chômage ${ }^{24}$. Des études d'observation ont constaté que l'assurance emploi ralentissait légèrement le retour au travail ${ }^{22}$. Les prestations de congé parental améliorent les paramètres de santé chez la mère et l'enfant, y compris les taux de mortalité néonatale, selon des études d'observation menées dans des pays à revenu élevé ${ }^{25,26}$. Les prestations de congé de maladie réduisent la propagation des maladies infectieuses et le stress ${ }^{27}$. L'élargissement des prestations d'assurance emploi durant la pandémie a bénéficié d'un solide appui ${ }^{28}$ qui perdurera sûrement.

Les critères d'admissibilité à l'assurance emploi ont été élargis durant la pandémie et cela pourrait se prolonger pendant la période de reprise postpandémique. Les barèmes d'assurance emploi qui existent déjà aux niveaux fédéral, provincial et territorial, peuvent être élargis et les critères d'admissibilité simplifiés. La durée des prestations de congé parental peut être prolongée. La durée des prestations de congé de maladie est actuellement déterminée par les employeurs dans la plupart des provinces et territoires; toutefois, cela pourrait être intégré plus généralement au marché de l'emploi parce que tous les travailleurs peuvent tomber malades. Ces formes de soutien pourraient être nécessaires en plus d'un revenu de subsistance universel.

Nous recommandons des formules de crédit ou de prêts abordables pour les personnes à revenu faible (très faible certitude des effets estimés).

L'accès au crédit et à de petits prêts abordables, sans intérêts ou à intérêts peu élevés, améliore plusieurs paramètres de santé chez les femmes et les enfants selon la revue systématique d'un essai et de 22 études quasi expérimentales effectuées dans des pays à revenu faible et moyen ${ }^{29}$ (p. ex., RC pour la mortalité infantile 0,78 , IC à $95 \% 0,68$ à $0,90^{30}$ ) (Annexe 1 , section $1 \mathrm{C})$. Certains programmes d'intervention en microfinancement (petits prêts) ont simultanément mis de l'avant d'autres mesures, comme la littératie médicale ${ }^{31}$. Même si ces interventions ont été conçues dans des milieux ruraux à faible revenu, elles ont aussi été mises en œuvre dans des contextes urbains à revenu élevé comme le quartier Queens, à New York, aux ÉtatsUnis $^{32}$. Le microfinancement repose sur une théorie selon laquelle les personnes à faible revenu pourraient tirer profit d'un accès à une forme de crédit qui leur est généralement refusée. Des études qualitatives auprès de populations spécifiques montrent que les programmes de microfinancement sont acceptés des bénéficiaires ${ }^{33}$.

Les « prêts sur salaire », à intérêts faibles et élevés qui doivent être remboursés rapidement, étaient fréquents au Canada avant la pandémie ${ }^{34,35}$. Dans d'autres pays, de petits prêts sont offerts par des organismes non gouvernementaux avec le soutien des gouvernements et un tel modèle fonctionnerait au Canada ${ }^{36}$.

\section{Logement}

Nous recommandons fortement l'élargissement des programmes de logements supervisés permanents étroitement liés à l'approche Logement d'abord, ayant les caractéristiques suivantes : engagement volontaire et gestion de cas, moins de $30 \%$ du revenu consacré au logement, choix du logement et des services de soutien, sans obligation de participation des bénéficiaires atteints de problèmes graves de santé mentale, éprouvant des problèmes de toxicomanie ou vivant en situation d'itinérance (forte certitude des effets estimés).

Les logements supervisés permanents améliorent la stabilité domiciliaire à long terme (> 5 ans) pour les personnes qui ont de graves problèmes de santé mentale ou qui éprouvent des problèmes de toxicomanie selon une revue systématique de 15 essais sur les logements supervisés permanents (risque relatif stabilité du logement à 6 ans, 1,42, IC à $95 \%$ 1,19 à 1,69) (voir Annexe 1 , section $2 A)^{37}$. Une autre revue systématique a mis au jour des bienfaits similaires ${ }^{38}$. Des essais randomisés et contrôlés (ERC) réalisés au Canada et aux É.U. en ont montré les 
bienfaits substantiels et les inconvénients minimes voire nuls ${ }^{37}$. La conformité des interventions à l'approche Logement d'abord mesurée sur une échelle à cet effet (Pathways Housing First Fidelity Scale) est associée à la stabilité domiciliaire ${ }^{39}$. Des études qualitatives confirment l'acceptabilité des logements supervisés permanents ${ }^{40,41}$.

Pendant la période de reprise postpandémique, les risques associés à l'itinérance continueront de poser une grave menace pour la santé, et la prévalence de l'itinérance sera probablement substantielle chez les individus atteints de grave maladie mentale. Élargir l'accès à des logements supervisés permanents pendant la période de reprise postpandémique pourrait favoriser grandement la stabilité du logement chez cette population au cours des prochaines années. En général, les logements supervisés permanents, et l'approche Logement d'abord en particulier, sont rentables ${ }^{42}$.

Nous recommandons d'élargir l'accès aux interventions de prévention des évictions, y compris l'accès à des services juridiques et à des conseils financiers (très faible certitude des effets estimés).

Les mesures axées sur la prévention des évictions, y compris des conseils financiers sur la façon de gérer les retards de loyer et des services parajuridiques ou juridiques, permettent de réduire le risque de recevoir un avis d'éviction, selon une revue systématique de 3 études, dont 1 essai et 2 études d'observation ${ }^{43}$. Par exemple, un ERC sur des personnes en attente de comparution pour retards de loyer devant l'équivalent d'une "régie du logement » de la ville de New York a révélé qu'une aide juridique gratuite réduisait les risques de remise d'un avis d'éviction (risque relatif 0,43, IC de $95 \% 0,30$ à 0,61) (Annexe 1, section 2B) ${ }^{44}$. La prévention des évictions est probablement acceptable pour les bénéficiaires; 1 essai a effectivement fait état d'un nombre similaire de comparutions, mais d'issues plus favorables ${ }^{44}$.

Maintenir les gens dans leur logement est une façon simple de prévenir l'itinérance. En raison de la pandémie, les gens ont eu plus de difficulté à s'y retrouver, car les programmes gouvernementaux se sont multipliés et les critères d'admissibilité ont été modifiés, alors que les services d'aide aux bénéficiaires de ces programmes étaient perturbés. La représentation juridique des locataires peut aider les gens à garder leur logement et à réduire les coûts associés à la résolution des différends et au non-paiement des loyers, tant pour les locataires que pour les propriétaires ${ }^{45}$.

\section{Violence conjugale}

Nous recommandons fortement des interventions qui incluent l'accès à l'aide juridique et des mesures de soutien aux victimes de violence conjugale (certitude modérée des effets estimés).

L'aide juridique et d'autres formes de soutien, telles que l'éducation et des thérapies pour les victimes de violence conjugale, réduisent la probabilité de violence physique dans certains contextes; elles atténuent le risque de dépression en plus d'améliorer le bien-être selon une revue systématique de 13 essais (p. ex., de brèves consultations juridiques ont réduit la violence physique mineure au bout de 1 an, différence moyenne
[DM] -1,0, IC à $95 \%-1,8$ à -0,2; des consultations juridiques intensives ont amélioré la qualité de vie des femmes vivant dans des refuges, DM 0,23, IC à $95 \% 0,00$ à 0,46) (Annexe 1 , section $3 A)^{46}$. Aucun effet négatif n'a été constaté lors de ces études, mais la crainte que les mesures juridiques n'attisent la violence est un obstacle potentiel ${ }^{47}$. Ce type d'intervention est acceptable pour les bénéficiaires, surtout en concertation avec les autres services nécessaires ${ }^{48}$.

Élargir ou permettre l'accès à l'aide juridique pourrait avoir des effets bénéfiques sur la santé en plus de réduire la violence conjugale, notamment en améliorant le logement et le revenu ${ }^{49}$. L'accès rapide, facile et gratuit à l'aide juridique sera important pour les personnes qui ont besoin de cette aide. Les interventions qui permettent aux victimes de violence conjugale de rester chez elles, comme le modèle australien Safe at home, ont fait l'objet d'études pilotes dans d'autres pays et pourraient être évaluées au Canada ${ }^{50}$.

Des interventions axées sur la toxicomanie et les traumatismes chez les agresseurs réduisent aussi le risque de violence selon une revue systématique de 17 études d'observation ${ }^{51}$. Ces études d'observation, effectuées surtout dans des pays à revenu élevé, ont révélé que les thérapies de groupe étaient associées à une réduction de la violence. Intervenir auprès des agresseurs et des victimes peut aussi réduire le risque de violence contre les femmes pendant la période de reprise postpandémique (Annexe 1 , section $3 \mathrm{C}$ ).

\section{Enfance}

Nous recommandons d'élargir l'accès à des services publics de garderie (faible certitude des effets estimés).

La fréquentation des services de garde peut améliorer le développement cognitif des enfants de moins de 5 ans selon 2 revues systématiques menées dans des pays à revenu élevé et faible, incluant chacun 1 essai (DM standardisée pour le développement cognitif 0,74 , IC à $95 \% 0,48$ à 1,00 dans les pays à revenu faible et moyen $^{52} ; 0,34$, IC à $95 \%-0,01$ à 0,69 dans les pays à revenu élevé ${ }^{53}$ ) (Annexe 1 , section $4 A$ ). Les autres bienfaits incluent de meilleures possibilités d'emploi pour les parents ${ }^{54}$. Les services publics de garderie seraient probablement très acceptables pour les familles, et surtout pour les parents qui souhaitent ou doivent travailler ${ }^{55}$.

Le nombre d'éducatrices et de garderies devra augmenter substantiellement pour accueillir les hausses d'inscription prévues. Mettre des garderies à la disposition des familles s'est révélé rentable lorsqu'on en a mesuré les avantages jusqu'à l'âge de 40 ans chez les enfants noirs ayant participé au programme HighScope Perry Preschool, instauré en 1962 au Michigan ${ }^{56}$.

Nous recommandons la distribution de nourriture saine aux enfants (certitude modérée des effets estimés).

La distribution de nourriture favorise le développement physique et psychomoteur des enfants de moins de 5 ans selon une revue systématique de 21 essais qui ont surtout été effectués dans des pays à revenu faible et moyen (augmentation du poids en 6 mois $0,12 \mathrm{~kg}$, IC à $95 \% 0,05$ à 0,18 ; DM 
standardisée pour le développement psychomoteur 0,41 , IC à $95 \% 0,10$ à 0,72 ) (Annexe 1 , section 4B) ${ }^{57}$. D'autres études menées dans des pays à revenu élevé ont révélé des bienfaits similaires selon le contexte, comme l'heure de la journée où les aliments étaient distribués et le type de supervision exercée durant les repas ${ }^{58}$.

Le recours aux banques alimentaires et l'insécurité alimentaire au Canada existaient déjà avant la pandémie ${ }^{59}$. La distribution de nourriture dans les écoles peut aider à améliorer la fréquentation scolaire ${ }^{60}$. La sélection des aliments doit se fonder sur les besoins diététiques des enfants et non sur la disponibilité des aliments donnés.

\section{Accès aux soins de santé}

Nous recommandons d'élargir l'accès au traitement de substitution aujourd'hui appelé " traitement par agoniste opioïde " (TAO) et aux sites d'injection supervisée (certitude modérée des effets estimés).

Le traitement par agoniste opioïde pour les problèmes liés à la consommation des opioïdes dans la population générale est associé à des réductions de l'utilisation d'opioïdes non prescrits selon une revue systématique de 11 essais (risque relatif $0,66, I C$ à $95 \% 0,56$ à 0,78 ) (Annexe 1 , section $5 A)^{61}$. Les sites d'injection supervisée réduisent les surdoses et facilitent l'accès aux soins (p. ex., réduction de $35 \%$ des surdoses fatales dans le voisinage immédiat d'un site d'injection supervisée) $)^{62}$, sans compter que l'accès aux soins médicaux pourrait réduire les préjudices ${ }^{63}$. Une méta-analyse de 28 études d'observation a révélé que le TAO est associé à une réduction de $50 \%$ du risque de nouveaux cas d'hépatite $C$ chez les utilisateurs de drogues par injection (risque relatif $0,50$, IC à $95 \% 0,40$ à 0,63$)^{64}$. L'utilisation combinée d'un programme d'échange de seringues et d'aiguilles et d'un TAO est associée à une réduction de $74 \%$ du risque d'hépatite $C$ (risque relatif 0,26, IC à $95 \% 0,07$ à 0,89$)^{64}$. Plusieurs études démontrent que le TAO est rentable et acceptable ${ }^{65,66}$. Le soutien aux sites d'injection supervisée varie, même si de nombreux Canadiens et Canadiennes connaissent quelqu'un qui pourrait en bénéficier ${ }^{67}$.

La pandémie et les perturbations qu'elle a entraînées sur le plan des soins de santé et des services sociaux, alliées à des stresseurs socio-économiques, ont probablement contribué à aggraver la crise des opioïdes ${ }^{68,69}$. Élargir l'accès au TAO et aux sites d'injection supervisée pourrait atténuer l'effet de la pandémie sur les surdoses, en plus d'exercer des effets durables en réduisant les obstacles actuels à l'amélioration des traitements à long terme. Ces mesures peuvent être combinées à d'autres interventions qui visent à sécuriser les approvisionnements, et réduire la stigmatisation et la judiciarisation des personnes toxicomanes et les effets néfastes de la toxicomanie ${ }^{70}$.

Nous recommandons d'élargir le dépistage du virus de l'immunodéficience humaine (VIH) et du virus de l'hépatite $C$ (VHC) aux personnes à haut risque (certitude modérée des effets estimés).
La pandémie a réduit l'accès aux services de dépistage du $\mathrm{VIH}$; le volume des tests a diminué pour plusieurs raisons, notamment : fermeture des sites de dépistage durant le confinement, réduction des effectifs, baisse de la participation et du nombre de rendez-vous attribués, laboratoires surchargés et réduction du nombre de demandes adressées aux établissements ${ }^{71}$. Élargir l'accès au dépistage du VIH et du VHC chez les personnes à haut risque pourrait réduire les effets néfastes à long terme des perturbations causées par la pandémie. Les consultations et tests volontaires rapides accroissent leur adoption (risque relatif 2,95, IC à 95\% 1,69 à 5,16 ) et la réception des résultats (risque relatif 2,14, IC à $95 \% 1,08$ à 4,24), comparativement aux tests classiques selon une revue systématique de 8 essais et 5 études d'observation (Annexe 1 , section $5 \mathrm{~B})^{72}$. Le dépistage du $\mathrm{VIH}$ et du VHC est recommandé chez les personnes à haut risque, y compris les personnes toxicomanes ${ }^{73}$. Les effets néfastes du dépistage pourraient inclure la stigmatisation et certains effets indésirables des traitements par ailleurs généralement bénéfiques ${ }^{72,74}$. Le dépistage s'est révélé acceptable pour les patients selon une revue exploratoire sur l'autodépistage du $\mathrm{VIH}$ qui incluait des études qualitatives et des essais ${ }^{74}$.

Élargir les programmes de dépistage serait probablement rentable selon une revue systématique de 26 études d'observation ${ }^{76}$.

Nous recommandons l'amélioration des soins de santé pour les personnes incarcérées (faible certitude des effets estimés).

Le traitement par agoniste opioïde durant l'incarcération réduit l'utilisation des opioïdes et accroît la participation aux soins selon une revue systématique de 22 essais et 21 études d'observation $^{77}$ (p. ex., résultat positif au dépistage urinaire des opioïdes $66 \%$ chez les témoins c. $25 \%$ avec la méthadone; nombre moyen de jours de traitement sur 1 année $23 \mathrm{j}$. pour les témoins c. $166 \mathrm{j}$. pour les patients sous méthadone $^{78}$ ) (Annexe 1 , section $5 \mathrm{C}$ ). Les avantages du traitement d'entretien pour la dépendance aux opioïdes dans les établissements de détention, comme la baisse d'utilisation de l'héroïne et des comportements à risque associés concordent avec les données probantes sur l'efficacité de ce traitement dans la collectivité ${ }^{79}$. Les interventions pharmacologiques ont des répercussions positives sur la mortalité, la toxicomanie, la fidélité au traitement et les facteurs criminogènes après la sortie de prison, si le traitement est administré durant l'incarcération et s'il est maintenu après ${ }^{79}$. Le TAO pour les personnes incarcérées est probablement acceptable et rentable ${ }^{80,81}$.

Il y a eu moins de dépistage du SRAS-CoV-2 dans les établissements de détention que dans les collectivités, même si les personnes incarcérées sont plus à risque; des taux élevés d'infection au SRAS-CoV-2 ont été observés dans la population carcérale ${ }^{7}$. Cela représente une occasion d'ajuster la prestation générale des soins de santé en fonction des besoins des personnes incarcérées, même si nous n'avons trouvé aucune étude sur la prestation générale des soins de santé dans cette population. 
Nous recommandons fortement d'intégrer les médicaments d'ordonnance dans le système public de santé au Canada (certitude modérée des effets estimés).

Éliminer ou réduire les coûts médicaux personnels assumés par les patients améliore l'observance thérapeutique et réduit l'utilisation des services de santé selon une revue systématique de 23 études d'observation ${ }^{82}$ (p. ex., augmentation des hospitalisations après dépassement des seuils imposés par les régimes d'assurance, risque relatif 1,85 , IC à $95 \% 1,64$ à $2,09^{83}$ ) (Annexe 1, section 5D). Un essai clinique au cours duquel on offrait leurs médicaments aux patients suivis en médecine de premier recours en Ontario quand ils n'avaient pas l'argent pour les payer a fait état d'une amélioration de l'observance thérapeutique et d'une baisse des coûts des soins de santé ${ }^{84}$. Les résultats d'une enquête représentative à l'échelle nationale montrent que le financement public des médicaments serait largement accepté ${ }^{85}$.

La pandémie a mis au jour une défaillance du système qui lie l'accès aux médicaments à l'occupation d'un emploi et à un régime d'assurance privé de l'employeur ou à la capacité des personnes de payer pour leurs médicaments quand des millions de Canadiens et de Canadiennes ont perdu leur emploi ou connu une baisse de revenu ${ }^{86}$. Les personnes racisées ont été affectées de manière disproportionnée ${ }^{85}$. Après un rapport d'un comité parlementaire de 2018 intitulé Régime d'assurance-médicaments universel pour tous les Canadiens : une nécessité, le Conseil consultatif sur la mise en œuvre d'un régime national d'assurance médicaments a étudié cette avenue ou l'inclusion des médicaments financés à même le système public de santé au Canada ${ }^{87,88}$. Le Bureau du directeur parlementaire du budget a estimé que la mise sur pied de l'assurance médicaments permettrait d'économiser 4 milliards de dollars par année ${ }^{89}$.

\section{Racisme}

Nous recommandons fortement une réflexion et des actions pour donner suite à de multiples rapports précédents qui proposent des approches pour lutter contre la discrimination à l'endroit des Autochtones, des personnes noires ou d'autres formes de racisme (énoncé non classé).

Avant la pandémie de COVID-19, plusieurs rapports ont décrit les effets néfastes du racisme, y compris à l'endroit des personnes noires et des Autochtones, et formulé des recommandations. Étant donné que le racisme affecte la santé, les recommandations de plusieurs documents touchant une vaste gamme d'enjeux sont pertinentes pour l'équité en matière de santé ${ }^{90-93}$ (Annexe 1, section 6). Notre recommandation est un énoncé non classé; elle repose sur l'importance de l'enjeu et sur la forte probabilité qu'elle procure des bienfaits, et elle s'appuie sur un vaste corpus de données indirectes connexes ${ }^{94}$. Il faut mener une réflexion sur la teneur des précédents rapports pour comprendre pourquoi si peu de choses ont été faites malgré les engagements successifs à lutter contre le racisme.

La Commission royale sur les peuples autochtones a présenté un rapport en 1996 dans lequel elle formulait plus de 100 recommandations allant du soutien aux enfants et aux adolescents jusqu'aux soins aux adultes âgés ${ }^{95}$. En 2014, le rapporteur spécial des Nations Unies sur les droits des peuples autochtones formulait 15 recommandations dans les catégories suivantes : conditions sociales et économiques, vérité et réconciliation, femmes et filles disparues, autonomie gouvernementale, participation et partenariat, négociations de traités et processus de revendication, et développement des ressources ${ }^{96}$.

Pour panser les plaies laissées par les pensionnats autochtones et aller de l'avant avec la réconciliation, en 2015, la Commission de Vérité et réconciliation a lancé 94 appels à l'action dans les domaines suivants : protection de l'enfance, éducation, langue et culture, santé, justice, Déclaration des Nations Unies sur les droits des peuples autochtones, proclamation et engagement (pacte) vis-à-vis de la réconciliation, convention de règlement, équité face au système de justice, Conseil national de réconciliation, développement et formation professionnels, excuses de l'Église, programmes pour les jeunes, musées et archives, renseignements sur les enfants disparus et leurs sépultures, commémoration, médias, sports, affaires et nouveaux arrivants au Canada ${ }^{95}$. En 2019, le rapport final de l'Enquête nationale sur les femmes et les filles autochtones disparues et assassinées a lancé 231 appels à la justice aux gouvernements, aux établissements, aux services sociaux, aux industries et à toute la population canadienne pour apporter des changements juridiques et sociaux qui pourront résoudre la crise nationale ${ }^{97}$. Quatre voies d'exploration ont été présentées : traumatisme historique multi- et intergénérationnel, marginalisation sociale et économique, maintien du statu quo et absence de volonté des institutions, et absence de prise en compte des capacités et de l'expertise des femmes, des filles et des personnes bispirituelles, lesbiennes, gaies, bisexuelles, transgenres, queer, en questionnement, intersexuelles et asexuelles (2SLGBTQQIA) autochtones ${ }^{97}$.

Le rapport de 2017 d'un groupe de travail des Nations Unies sur les droits de la personne a formulé 15 recommandations liées au racisme envers les personnes noires dans le système de justice criminelle; crimes haineux; éducation; santé; logement et emploi; et intersectionnalité de la discrimination ${ }^{98}$. Dans un rapport de 1992 adressé au premier ministre de l'Ontario au sujet du racisme à l'endroit des personnes noires, on formulait 23 recommandations et on insistait sur la nécessité de mettre en œuvre les recommandations des décennies précédentes, tout en admettant un certain " effarement " de constater que certaines d'entre elles n'étaient toujours pas mises en œuvre ${ }^{99}$. Plusieurs rapports avaient insisté sur le racisme dans les soins de santé, y compris les rapports sur les données compilées par Well Living House ${ }^{100}$.

Malgré les recommandations existantes, le racisme systémique a eu des conséquences disproportionnées au cours de la pandémie sur les personnes racisées, comme en témoignent les taux plus élevés d'infection au SRAS-CoV-2 et de décès ${ }^{3,101}$. Le racisme n'est pas nouveau, ni les appels urgents à l'action. La reprise postpandémique serait un moment approprié pour enfin mettre en œuvre les recommandations passées afin de lutter efficacement contre le racisme et promouvoir la santé. 


\section{Méthodes}

Nous avons utilisé l'approche GRADE (Grading of Recommendations Assessment, Development and Evaluation) pour formuler nos recommandations et nous avons suivi le guide AGREE II (Appraisal of Guidelines for Research and Evaluation) pour rédiger notre rapport ${ }^{11,102}$.

\section{Composition du Comité des recommandations}

Le Centre MAP pour des solutions de santé urbaine est un centre de recherche qui s'intéresse à l'équité en matière de santé. Il est rattaché l'un des sites du réseau hospitalier Unity Health Toronto, l'hôpital St. Michael et est affilié à part entière à l'Université de Toronto. Étant donné la raison d'être spécifique du centre, nous avons formé le Comité des recommandations en envoyant des invitations par courriel à tous les chercheurs du centre en août 2020; parmi les chercheurs invités, 8 sur 28 (29\%) ont décidé d'y participer. Les champs d'expertise des membres de notre comité sont les suivants : science de la mise en œuvre, revenu, logement, services de santé, médecine de premiers recours et discrimination.

Nous avons mobilisé des membres du public $(n=4)$, y compris des personnes ayant une expérience concrète des iniquités en matière de santé, autour des thèmes et des recommandations à l'occasion de 2 téléconférences en août 2020 et février 2021.

\section{Choix des thèmes}

Une première liste de thèmes a été préparée (par N.P.) à partir de rapports publiés au sujet des iniquités en matière de santé au Canada ${ }^{103,104}$ et de discussions avec des personnes défavorisées, et nous l'avons distribuée par l'entremise d'un sondage en ligne à tous les chercheurs du centre $(n=28)$ qui ont été invités à suggérer d'autres thèmes ou à réviser les thèmes proposés $(n=7$ [25\%] ont répondu).

Les thèmes finaux ont ensuite été sélectionnés lors d'une réunion du Comité des recommandations et 6 thèmes (revenu, logement, violence conjugale, enfance, racisme et accès aux soins de santé [incluant 4 sous-thèmes]) ont été retenus par voie de consensus en tenant compte de la contribution de chaque facteur à des iniquités évitables sur le plan des paramètres de santé. Nous avons procédé à des interrogations préliminaires de la documentation sur tous les thèmes suggérés, mais nous ne les avons pas toutes approfondies.

\section{Revue documentaire}

Nous avons procédé à des interrogations documentaires sur les populations, les interventions, les comparateurs et les paramètres choisis pour chaque thème en collaboration avec une bibliothécaire des sciences de la santé. Nous (H.W., A.W. et I.A.) avons interrogé le réseau Ovid MEDLINE pour trouver des revues systématiques révisées par des pairs sur les paramètres de santé publiées en langue anglaise entre le $1^{\text {er }}$ janvier 2000 et

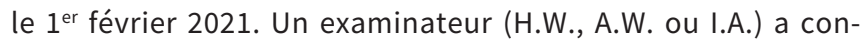
firmé la pertinence de chaque revue systématique et son opinion a été confirmée ou débattue par un second examinateur.
Les désaccords ont été résolus par voie de discussion ou de consultation avec un tiers réviseur (H.W., A.W., I.A. ou N.P.). Nous avons effectué des recherches séparées sur les préférences et les valeurs relatives à chaque thème.

\section{Élaboration de recommandations}

Pour chaque thème, un cadre a été établi afin de passer des données probantes au libellé d'une décision (Annexe 1, sections $1-6)^{105}$. Les membres du Comité des recommandations ont passé ces cadres en revue et rédigé l'ébauche des recommandations, en tenant compte des préférences et des valeurs selon les résultats des interrogations documentaires pertinentes.

Le Comité des recommandations a tenu une série de réunions virtuelles en janvier et février 2021 (les 6, 13 et 17 janvier et les 10 et 24 février) pour asseoir les recommandations finales et établir la solidité de chacune et la certitude de leurs effets estimés par voie de consensus. Conformément à l'approche GRADE, nous avons formulé des recommandations fortes (« nous recommandons fortement ») et faibles (" nous recommandons ») et nous avons classé séparément les décisions quant à la force des recommandations et quant à la certitude de leurs effets estimés ${ }^{11}$. Pour un thème (racisme), nous avons décidé de formuler un énoncé non classé en raison de l'importance de l'enjeu, de la forte probabilité de bienfait de la recommandation et de l'appui d'un imposant corpus de données connexes indirectes ${ }^{94}$. Toutes les recommandations ont été préparées par consensus et aucun vote n'a été nécessaire.

\section{Revue externe}

Nous avons partagé l'ébauche des recommandations avec des personnes ayant une expérience concrète $(n=4)$ ainsi que certains décideurs $(n=3)$ et représentants d'organismes nationaux liés à la pratique clinique et à la santé publique $(n=19$; Annexe 1, section 7D), pour une contribution plus diversifiée venant de parties prenantes de différents horizons. Nous avons sollicité leurs commentaires au moyen de questions ouvertes et intégré leurs suggestions après que le Comité des recommandations les ait passées en revue. Les modifications consécutives à la revue externe ont inclus une reformulation des recommandations et des modifications aux textes explicatifs, notamment des questions liées à la mise en œuvre des recommandations.

\section{Gestion des intérêts concurrents}

Nous avons géré les intérêts concurrents conformément aux principes du Guidelines International Network, sous la direction d'un comité de surveillance externe des conflits d'intérêts ${ }^{106}$. Le Comité des recommandations se composait d'individus qui n'avaient aucun conflit d'intérêts directs, conformément aux directives ${ }^{107}$. Les membres du Comité ont été invités à signaler tout intérêt concurrent potentiel, y compris des intérêts concurrents indirects; le Comité était composé d'individus qui avaient des intérêts à divers niveaux, ce qui a limité les effets potentiels des conflits d'intérêts intellectuels ${ }^{107}$. 


\section{Mise en œuvre}

Le Centre MAP pour des solutions en santé urbaine tient un registre des documents à l'appui des recommandations sur son site Web (maphealth.ca). Nous allons maintenir une liste des ressources connexes avec la ligne directrice, à partir des suggestions de diverses parties prenantes consultées. Nous avons partagé la ligne directrice avec des décideurs clés aux niveaux fédéral, provincial et municipal (Annexe 1, section 7D).

L'attention publique actuelle pour les iniquités durant la pandémie, alliée à l'engagement du gouvernement du Canada à réduire la pauvreté de $50 \%$ d'ici 2030 , pourrait faciliter des changements pérennes ${ }^{108}$. Les obstacles à la mise en œuvre des changements incluent une baisse l'intérêt à l'endroit des iniquités à mesure que les risques directs de la pandémie se dissipent pour la majorité des Canadiens et Canadiennes, l'influence de celles et ceux qui tirent profit d'un statu quo inéquitable (p. ex., les compagnies qui profitent du fait que certains médicaments soient exclus des listes des régimes de santé publics) et le désir de passer à autre chose une fois la pandémie résolue.

Les recommandations doivent être mises en œuvre de manière équitable. Par exemple, les initiatives visant à améliorer l'accès à des logements supervisés permanents devraient aussi se préoccuper d'autres formes d'iniquités, comme le racisme. Nous prévoyons détailler certaines des recommandations au sujet de leur mise en œuvre en collaboration avec d'autres instances, y compris des organismes qui offrent des services connexes et qui mènent des actions de représentation sur ces enjeux.

Nous prévoyons étudier l'utilité de mettre ces recommandations à jour dans 3 ans et d'afficher les mises à jour sur le site www.maphealth.ca. On peut suivre la mise en œuvre des recommandations en mesurant les taux de pauvreté, le nombre de prêts accordés à des personnes à revenu faible, la proportion d'individus éprouvant de graves problèmes de santé mentale qui n'ont pas accès à des logements supervisés permanents, les taux globaux d'hébergement, les taux d'éviction, les taux de violence conjugale, le soutien aux victimes de violence conjugale, le nombre d'enfants en garderie, la sécurité alimentaire des enfants, les taux de mortalité liés aux opioïdes, les taux d'utilisation d'un TAO et des sites d'injections supervisées, l'incidence et le fardeau du VIH et du VHC, la santé et l'accès aux soins de santé pour les détenus, la piètre observance thérapeutique liée au coût des médicaments, les paramètres de santé (y compris la mortalité) chez les personnes racisées et les progrès vers la mise en œuvre des recommandations existantes en lien avec la discrimination envers les Autochtones et autres formes de racisme. Le suivi pourrait être effectué par des agences fédérales, provinciales et territoriales, comme l'Agence de la santé publique du Canada, avec la participation d'autres instances, notamment les fournisseurs de services et les chercheurs. Des changements de politiques peuvent créer de nouvelles iniquités; il est donc important de mesurer régulièrement divers paramètres.

\section{Autres lignes directrices}

Le rapport de l'Administratrice en chef de la santé publique du Canada, Du risque à la résilience : Une approche axée sur l'équité concernant la COVID-19, décrit des approches qui recoupent nos recommandations, y compris l'approche Logement d'abord, la réduction des préjudices liés aux opioïdes et la prévention de la violence conjugale ${ }^{1}$. Le rapport intitulé Build Back Fairer présente les stratégies de haut niveau pour corriger les iniquités en Angleterre dans la foulée de la pandémie de COVID-19 ${ }^{109}$. D'autres groupes ont formulé des recommandations en lien avec la période de reprise postpandémique au sujet de thèmes spécifiques tels que le logement ${ }^{110}$, les initiatives de défense des intérêts visant une "reprise pour tous » (recoveryforall.ca). Avant la pandémie, une ligne directrice a formulé une forte recommandation similaire en faveur des logements supervisés permanents ${ }^{111}$.

\section{Connaissances à parfaire}

Pour certaines modifications ou interventions, nous disposions de renseignements limités sur les ressources nécessaires à leur mise en œuvre, leur acceptabilité, leur faisabilité et leur rapport coût-efficacité. Des interventions, comme les transferts de fonds, ont été étudiées surtout dans des pays à revenu faible et moyen, même si elles sont tout aussi pertinentes pour les pays à revenu élevé. Les interventions ont été sélectionnées sur la base de leur capacité à promouvoir l'équité, mais on ignore si concrètement, elles peuvent être mises en œuvre de façon juste et équitable.

\section{Limites de la présente ligne directrice}

Les thèmes et les questions de recherche ont été sélectionnés par les membres du Comité, qui sont tous des chercheurs; d'autres groupes auraient pu choisir des thèmes différents et formuler d'autres recommandations pour la promotion de l'équité en matière de santé. Nos recommandations visant l'équité en matière de santé ne devraient pas être perçues comme exhaustives ou définitives. Nous insistons parfois sur les services aux individus (p. ex., prévention des évictions) plutôt que sur des changements systémiques (p. ex., bannissement des évictions), même si d'autres approches auraient pu générer des recommandations différentes selon les études disponibles. Certains thèmes, comme la santé des travailleurs migrants ou les soins de longue durée dans les réseaux publics-privés n'ont pas été explicitement inclus, alors que nous en reconnaissons l'importance. D'autres thèmes importants, comme la santé des enfants autochtones, ont été inclus indirectement, alors qu'ils auraient pu mener à plusieurs recommandations indépendantes, comme c'est le cas dans certains documents cités, notamment dans le rapport final de la Commission de vérité et réconciliation où l'on retrouve 5 appels à l'action à cet égard ${ }^{95}$.

Des parties prenantes ont suggéré d'autres thèmes, comme la santé mentale des enfants; nous en avons tenu compte et effectué des interrogations de la documentation, mais décidé de ne pas aller de l'avant lorsqu'il n'était pas clair que les 
interventions étudiées allaient promouvoir l'équité en matière de santé. De grands enjeux comme l'itinérance ne seront pas résolus par la simple mise en œuvre de nos quelques recommandations axées sur des populations spécifiques; d'autres efforts s'imposent et certains des changements requis ne s'appuient sur aucune étude.

Nous nous sommes fiés à des revues systématiques effectuées avant la pandémie, dans la plupart des cas, et elles peuvent avoir exclu des études importantes publiées avant et durant la pandémie. Certaines des interventions n'ont été étudiées que dans des contextes spécifiques et peuvent avoir été étudiées séparément, alors que nos recommandations sont interreliées (p. ex., amélioration de la sécurité du revenu permettant une meilleure stabilité domiciliaire).

\section{Conclusion}

La période de reprise postpandémique est l'occasion de s'attaquer aux iniquités en matière de santé qui ont mené à une distribution inéquitable du fardeau et des effets néfastes de la COVID-19. Des changements de politiques aux niveaux fédéral, provincial et municipal qui favorisent l'équité en matière de santé avaient été étudiés avant cette pandémie et devraient être mis en œuvre avant la suivante. Ces changements concordent avec les appels à la lutte contre la pauvreté qui datent de l'époque prépandémique et toute l'attention accordée aux iniquités durant la pandémie pourrait faciliter ces changements. Leurs effets interreliés sur l'équité en matière de santé doivent être mesurés soigneusement pour orienter les politiques futures au Canada et ailleurs.

\section{Références}

1. From risk to resilience: an equity approach to COVID-19: the Chief Public Health Officer of Canada's report on the state of public health in Canada 2020. Ottawa: Public Health Agency of Canada; 2020.

2. Enhanced epidemiological summary: COVID-19 in Ontario - a focus on material deprivation. Toronto: Public Health Ontario:1-24.

3. Enhanced epidemiological summary: COVID-19 in Ontario - A Focus on Diversity. Toronto: Public Health Ontario; 2020:1-22.

4. Populations disproportionately impacted by COVID-19: current state assessment. City of Vancouver, Social Policy and Projects Research Team; 2021:1-73.

5. Richard L, Booth R, Rayner J, et al. Testing, infection and complication rates of COVID-19 among people with a recent history of homelessness in Ontario, Canada: a retrospective cohort study. CMAJ Open 2021;9:E1-9.

6. Gomes T, Kitchen SA, Murray R. Measuring the burden of opioid-related mortality in Ontario, Canada, during the COVID-19 pandemic. JAMA Netw Open 2021;4:e2112865

7. Blair A, Parnia A, Siddiqi A. A time-series analysis of testing and COVID-19 outbreaks in Canadian federal prisons to inform prevention and surveillance efforts. Can Commun Dis Rep 2021;47:66-76.

8. Pandemic influenza preparedness and response: a WHO guidance document. Geneva: World Health Organization; 2009. Accessible ici : https://www.ncbi. nlm.nih.gov/books/NBK143062/ (consulté le 3 mars 2021).

9. Best Brains Exchange proceedings report: Strengthening the structural determinants of health post-COVID-19. Ottawa: Public Health Agency of Canada; 2020; modified 2021 Mar. 16. Accessible ici : https://www.canada.ca/en/public-health/corporate/ publications/chief-public-health-officer-reports-state-public-health-canada/from -risk-resilience-equity-approach-covid-19/best-brains-exchange-proceedings-report .html (consulté le 9 avr. 2021).
10. COVID-19 in Canada: a one-year update on social and economic impacts. Cat no 11-631-x2021001. Ottawa: Statistics Canada; 2021 Mar. 11:1-52. Accessible ici : https://www150.statcan.gc.ca/n1/pub/11-631-x/11-631-x2021001-eng.htm (consulté le 12 nov. 2021).

11. Schünemann H, Brożek J, Guyatt G, et al., editors. GRADE handbook. London (UK): GRADE Working Group; 2013. Accessible ici : https://gdt.gradepro.org/ app/handbook/handbook.html (consulté le 4 mars 2021).

12. Pega F, Liu SY, Walter S, et al. Unconditional cash transfers for reducing poverty and vulnerabilities: effect on use of health services and health outcomes in lowand middle-income countries. Cochrane Database Syst Rev 2017;11:CD011135.

13. Results of Finland's basic income experiment: small employment effects, better perceived economic security and mental wellbeing. Helsinki (Finland): Kela; 2020. Accessible ici : https://www.kela.fi/web/en/news-archive/-/asset_publisher/ IN08GY2nIrZo/content/results-of-the-basic-income-experiment-small-employment -effects-better-perceived-economic-security-and-mental-wellbeing (consulté le 3 mars 2021).

14. Salehi-Isfahani D, Mostafavi-Dehzooei MH. Cash transfers and labor supply: evidence from a large-scale program in Iran. J Dev Econ 2018;135:349-67.

15. Forget EL. New questions, new data, old interventions: the health effects of a guaranteed annual income. Prev Med 2013;57:925-8.

16. Baker AC, Martin-West S, Samra S, et al. Mitigating loss of health insurance and means tested benefits in an unconditional cash transfer experiment: implementation lessons from Stockton's guaranteed income pilot. SSM Popul Health 2020;11:100578.

17. Williams CE. Taking bold action on homelessness. Foundations for social change: New Leaf project. Vancouver: Foundations for Social Change; 2020:1-17. Accessible ici : https://static1.squarespace.com/static/5f07a92f21d34b403c788e05 /t/617989f9898f760c97fcbace/1635355131600/2021_FSC_Statement_of_Impact _W_Expansion.pdf (consulté le 12 nov. 2021).

18. Delsen L, Schilpzand R. Individual preferences for the unconditional basic income in the European Union. In: Delsen L, editor. Empirical Research on an Unconditional Basic Income in Europe. Cham: Springer; 2019:29-60.

19. Mendelson M. Lessons from Ontario's basic income pilot. Maytree; 2019. Accessible ici : https://www.deslibris.ca/ID/10102735 (consulté le 3 mars 2021).

20. Table 11-10-0066-01: Market Basket Measure (MBM) thresholds for the reference family by Market Basket Measure region, component and base year. Ottawa: Statistics Canada; modified 2021 Oct. 27. Accessible ici : https:// www150.statcan.gc.ca/t1/tbl1/en/tv.action?pid=1110006601 (consulté le 3 mars 2021).

21. Ammar N, Busby C, Ahmed SM. Distributional and fiscal analysis of a national guaranteed basic income. Ottawa: Office of the Parliamentary Budget Officer; 2021. Accessible ici : https://www.pbo-dpb.gc.ca/en/blog/news/RP-2122-001 -S--distributional-fiscal-analysis-national-guaranteed-basic-income--analyse -financiere-distributive-un-revenu-base-garanti-echelle-nationale (consulté le 9 avr. 2021).

22. Renahy E, Mitchell C, Molnar A, et al. Connections between unemployment insurance, poverty and health: a systematic review. Eur J Public Health 2018;28:269-75.

23. Virtanen $\mathrm{P}$, Liukkonen $\mathrm{V}$, Vahtera J, et al. Health inequalities in the workforce: the labour market core-periphery structure. Int J Epidemiol 2003;32:1015-21.

24. O'Campo P, Molnar A, Ng E, et al. Social welfare matters: a realist review of when, how, and why unemployment insurance impacts poverty and health. Soc Sci Med 2015;132:88-94.

25. Jou J, Kozhimannil KB, Abraham JM, et al. Paid maternity leave in the United States: associations with maternal and infant health. Matern Child Health $J$ 2018;22:216-25.

26. Khan MS. Paid family leave and children health outcomes in OECD countries. Child Youth Serv Rev 2020;116:105259.

27. Piper K, Youk A, James AE III, et al. Paid sick days and stay-at-home behavior for influenza. PLoS One 2017;12:e0170698.

28. Canadians' opinions on possible key priorities for the federal budget. Project 2016-798. CTV News/NANOS Survey. NANOS Research; 2016:1-17. Accessible ici : https://www.nanos.co/wp-content/uploads/2017/07/2016-798A-CTV-February -Budget-and-the-Economy-Populated-Report-w-tabs-R.pdf (consulté le 12 nov. 2021). 
29. Orton L, Pennington A, Nayak S, et al. Group-based microfinance for collective empowerment: a systematic review of health impacts. Bull World Health Organ 2016;94:694-704A.

30. Bhuiya A, Chowdhury M. Beneficial effects of a woman-focused development programme on child survival: evidence from rural Bangladesh. Soc Sci Med 2002;55:1553-60.

31. Lorenzetti LMJ, Leatherman S, Flax VL. Evaluating the effect of integrated microfinance and health interventions: an updated review of the evidence. Health Policy Plan 2017;32:732-56.

32. Financial information. Washington (DC): Grameen Foundation. Accessible ici : https:/grameenfoundation.org/partners/resources/financial-information (consulté le 3 mars 2021).

33. Lall P, Shaw SA, Saifi R, et al. Acceptability of a microfinance-based empowerment intervention for transgender and cisgender women sex workers in Greater Kuala Lumpur, Malaysia. J Int AIDS Soc 2017;20:21723.

34. Payday loans: market trends. Ottawa: Financial Consumer Agency of Canada; 2016:1-20. Accessible ici : https://www.canada.ca/en/financial-consumer-agency/ programs/research/payday-loans-market-trends.html (consulté le 12 nov. 2021).

35. Dijkema B. The changing face of payday lending in Canada. Hamilton (ON): Cardus; 2019. Accessible ici : https://www.cardus.ca/research/work-economics/reports/ the-changing-face-of-payday-lending-in-canada/ (consulté le 12 nov. 2021).

36. Khandker SR, Khalily MAB, Samad HA. Beyond ending poverty: the dynamics of microfinance in Bangladesh. Washington (DC): World Bank; 2016. Accessible ici : https://openknowledge.worldbank.org/handle/10986/24669 (consulté le 12 nov. 2021).

37. Aubry T, Bloch G, Brcic V, et al. Effectiveness of permanent supportive housing and income assistance interventions for homeless individuals in high-income countries: a systematic review. Lancet Public Health 2020;5:e342-60.

38. Hwang SW, Tolomiczenko G, Kouyoumdjian FG, et al. Interventions to improve the health of the homeless: a systematic review. Am J Prev Med 2005;29:311-9.

39. Goering P, Veldhuizen S, Nelson GB, et al. Further validation of the pathways housing first fidelity scale. Psychiatr Serv 2016;67:111-4.

40. Nelson G, Patterson M, Kirst M, et al. Life changes among homeless persons with mental illness: a longitudinal study of housing first and usual treatment. Psychiatr Serv 2015;66:592-7.

41. Piat M, Seida K, Padgett D. Choice and personal recovery for people with serious mental illness living in supported housing. J Ment Health 2020;29:306-13.

42. Latimer EA, Rabouin D, Cao Z, et al.; At Home/Chez Soi Investigators. Costeffectiveness of housing first with assertive community treatment: results from the Canadian At Home/Chez Soi trial. Psychiatr Serv 2020;71:1020-30.

43. Holl M, van den Dries L, Wolf JRLM. Interventions to prevent tenant evictions: a systematic review. Health Soc Care Community 2016;24:532-46.

44. Seron C, Ryzin GG, Frankel MM, et al. The impact of legal counsel on outcomes for poor tenants in New York City's housing court: results of a randomized experiment. Law Soc Rev 2001;35:419-34.

45. Evans G. AcAteer M. Does debt advice pay? A business case for social landlords final report. London (UK): The Financial Inclusion Centre; 2011:1-93. Accessible ici : http://inclusioncentre.co.uk/wordpress29/wp-content/uploads/2011/12/ Does_Debt-Advice_Pay_A-Business_Case_for_Social_Landlords_Final_Report. pdf (consulté le 12 nov. 2021).

46. Rivas C, Ramsay J, Sadowski L, et al. Advocacy interventions to reduce or eliminate violence and promote the physical and psychosocial well-being of women who experience intimate partner abuse. Cochrane Database Syst Rev 2015;(12):CD005043.

47. Rodriguez MA, Bauer HM, McLoughlin E, et al. Screening and intervention for intimate partner abuse: practices and attitudes of primary care physicians. JAMA 1999;282:468-74.

48. Wadsworth P, Kothari C, Lubwama G, et al. Health and health care from the perspective of intimate partner violence adult female victims in shelters: impact of IPV, unmet needs, barriers, experiences, and preferences. Fam Community Health 2018;41:123-33.

49. Martinez O, Boles J, Muñoz-Laboy M, et al. Bridging health disparity gaps through the use of medical legal partnerships in patient care: a systematic review. J Law Med Ethics 2017;45:260-73.
50. Edwards R. Staying home, leaving violence: promoting choices for women leaving abusive partners. Sydney (AU): Australian Domestic and Family Violence Clearinghouse; 2004.

51. Karakurt G, Koç E, Çetinsaya EE, et al. Meta-analysis and systematic review for the treatment of perpetrators of intimate partner violence. Neurosci Biobehav Rev 2019;105:220-30.

52. Brown TW, van Urk FC, Waller R, et al. Centre-based day care for children younger than five years of age in low- and middle-income countries. Cochrane Database Syst Rev 2014;(9):CD010543.

53. van Urk FC, Brown TW, Waller R, et al. Centre-based day care for children younger than five years of age in high-income countries. Cochrane Database Syst Rev 2014;(9):CD010544.

54. Toroyan T, Roberts I, Oakley A, et al. Effectiveness of out-of-home day care for disadvantaged families: randomised controlled trial. BMJ 2003;327:906.

55. Carlin C, Davis EE, Krafft C, et al. Parental preferences and patterns of child care use among low-income families: a Bayesian analysis. Child Youth Serv Rev 2019;99:172-85.

56. Muennig P, Schweinhart L, Montie J, et al. Effects of a prekindergarten educational intervention on adult health: 37-year follow-up results of a randomized controlled trial. Am J Public Health 2009;99:1431-7.

57. Kristjansson E, Francis DK, Liberato S, et al. Food supplementation for improving the physical and psychosocial health of socio-economically disadvantaged children aged three months to five years. Cochrane Database Syst Rev 2015;2015:CD009924.

58. Colley P, Myer B, Seabrook J, et al. The impact of Canadian school food programs on children's nutrition and health: a systematic review. Can J Diet Pract Res 2019;80:79-86.

59. Hunger Report 2020: the impact of COVID-19 on food bank use in Ontario. Toronto: Feed Ontario; 2020. Accessible ici : https://feedontario.ca/hunger -report-2020/ (consulté le 3 mars 2021).

60. Grantham-McGregor S. Can the provision of breakfast benefit school performance? Food Nutr Bull 2005;26(Suppl 2):S144-58.

61. Mattick RP, Breen C, Kimber J, et al. Methadone maintenance therapy versus no opioid replacement therapy for opioid dependence. Cochrane Database Syst Rev 2009;(3):CD002209.

62. Marshall BDL, Milloy M-J, Wood E, et al. Reduction in overdose mortality after the opening of North America's first medically supervised safer injecting facility: a retrospective population-based study. Lancet 2011;377:1429-37.

63. Magwood O, Salvalaggio G, Beder M, et al. The effectiveness of substance use interventions for homeless and vulnerably housed persons: a systematic review of systematic reviews on supervised consumption facilities, managed alcohol programs, and pharmacological agents for opioid use disorder. PLOS One 2020;15:e0227298.

64. Platt L, Minozzi S, Reed J, et al. Needle and syringe programmes and opioid substitution therapy for preventing HCV transmission among people who inject drugs: findings from a Cochrane Review and meta-analysis. Addiction 2018;113:545-63.

65. Larance B, Degenhardt L, Grebely J, et al. Perceptions of extended-release buprenorphine injections for opioid use disorder among people who regularly use opioids in Australia. Addiction 2020;115:1295-305.

66. Lange BCL, Bach-Mortensen AM. A systematic review of stakeholder perceptions of supervised injection facilities. Drug Alcohol Depend 2019;197:299-314.

67. Opioids in Canada: one-in-eight have family or close friends who faced addiction. Vancouver: Angus Reid Institute; 2018. Accessible ici : https://angusreid. org/opioid-crisis/ (consulté le 28 sept. 2021).

68. Ontario Drug Policy Research Network; Office of the Chief Coroner for Ontario/ Ontario Forensic Pathology Service; Public Health Ontario; Centre on Drug Policy Evaluation. Preliminary patterns in circumstances surrounding opioid-related deaths in Ontario during the COVID-19 pandemic. Toronto: Public Health Ontario; 2020:1-24. Accessible ici : https://www.publichealthontario.ca/-/media/ documents/o/2020/opioid-mortality-covid-surveillance-report.pdf?la=en (consulté le 12 nov. 2021).

69. Illicit drug toxicity deaths in BC: January 1, 2011-January 31, 2021. Burnaby (BC): Office of the Chief Coroner; 2021:1-26. Accessible ici : https://www2.gov.bc.ca/ assets/gov/birth-adoption-death-marriage-and-divorce/deaths/coroners-service/ statistical/illicit-drug.pdf (consulté le 12 nov. 2021). 
70. Norton A, Kerr T. Applying the lessons of COVID-19 response to Canada's worsening opioid epidemic. EClinicalMedicine 2020;29:100633.

71. Simões D, Stengaard AR, Combs L, et al.; EuroTEST COVID-19 impact assessment consortium of partners. Impact of the COVID-19 pandemic on testing services for HIV, viral hepatitis and sexually transmitted infections in the WHO European Region, March to August 2020. Euro Surveill 2020;25:2001943.

72. Pottie K, Medu O, Welch V, et al. Effect of rapid HIV testing on HIV incidence and services in populations at high risk for HIV exposure: an equity-focused systematic review. BMJ Open 2014;4:e006859.

73. Grad R, Thombs B, Tonelli M, et al.; Canadian Task Force on Preventive Health Care. Recommendations on hepatitis C screening for adults. CMAJ 2017; 189:E594-604.

74. Chou R, Dana T, Grusing S, et al. Screening for HIV infection in asymptomatic, nonpregnant adolescents and adults: updated evidence report and systematic review for the US Preventive Services Task Force. JAMA 2019;321:2337-48.

75. Hawk ME, Chung A, Creasy SL, et al. A scoping review of patient preferences for HIV self-testing services in the United States: implications for harm reduction. Patient Prefer Adherence 2020;14:2365-75.

76. Bert F, Gualano MR, Biancone P, et al. Cost-effectiveness of HIV screening in high-income countries: a systematic review. Health Policy 2018;122:533-47.

77. Malta M, Varatharajan T, Russell C, et al. Opioid-related treatment, interventions, and outcomes among incarcerated persons: a systematic review. PLoS Med 2019;16:e1003002.

78. Kinlock TW, Gordon MS, Schwartz RP, et al. A randomized clinical trial of methadone maintenance for prisoners: results at 12 months postrelease. J Subst Abuse Treat 2009;37:277-85.

79. Hedrich D, Alves P, Farrell M, et al. The effectiveness of opioid maintenance treatment in prison settings: a systematic review. Addiction 2012;107:501-17.

80. Larney S, Zador D, Sindicich N, et al. A qualitative study of reasons for seeking and ceasing opioid substitution treatment in prisons in New South Wales, Australia. Drug Alcohol Rev 2017;36:305-10.

81. Horn BP, Li X, Mamun S, et al. The economic costs of jail-based methadone maintenance treatment. Am J Drug Alcohol Abuse 2018;44:611-8.

82. Kesselheim AS, Huybrechts KF, Choudhry NK, et al. Prescription drug insurance coverage and patient health outcomes: a systematic review. Am J Public Health 2015;105:e17-30.

83. Raebel MA, Delate T, Ellis JL, et al. Effects of reaching the drug benefit threshold on Medicare members' healthcare utilization during the first year of Medicare Part D. Med Care 2008;46:1116-22.

84. Persaud N, Bedard M, Boozary A, et al. Adherence at 2 years with distribution of essential medicines at no charge: The CLEAN Meds randomized clinical trial. PLoS Med. 2021 May 1;18(5):e1003590.

85. Access for all: near universal support for a pharmacare plan covering Canadians' prescription drug costs. Vancouver: Angus Reid Institute; 2020. Accessible ici : https://angusreid.org/pharmacare-2020/ (consulté le 3 mars 2021).

86. Labour Force Survey, January 2021. Ottawa: Statistics Canada; 2021, modified 2021 Feb. 5. Accessible ici : https://www150.statcan.gc.ca/n1/daily-quotidien/210205/ dq210205a-eng.htm (consulté le 4 mars 2021).

87. Casey B. Pharmacare now: prescription medicine coverage for all Canadians. 42nd Parliament, 1st sess. Ottawa: House of Commons; 2018:1-140.

88. Advisory Council on the Implementation of National Pharmacare. Ottawa: Health Canada; modified 2018 July 2. Accessible ici : https://www.canada.ca/ en/health-canada/corporate/about-health-canada/public-engagement/external -advisory-bodies/implementation-national-pharmacare.html (consulté le 3 mars 2021).

89. Malanik C. Federal cost of a national pharmacare program. Ottawa: Office of the Parliamentary Budget Officer; 2017. Accessible ici : https://www.pbo-dpb. gc.ca/en/blog/news/Pharmacare (consulté le 3 mars 2021).

90. Paradies Y, Ben J, Denson N, et al. Racism as a determinant of health: a systematic review and meta-analysis. PLoS One 2015;10:e0138511.

91. Gee GC, Ford CL. Structural racism and health inequities: old issues, new directions. Du Bois Rev 2011;8:115-32.
92. Williams DR, Cooper LA. Reducing racial inequities in health: using what we already know to take action. Int J Environ Res Public Health 2019;16:606.

93. Bailey ZD, Krieger N, Agénor M, et al. Structural racism and health inequities in the USA: evidence and interventions. Lancet 2017;389:1453-63.

94. Guyatt GH, Alonso-Coello P, Schünemann HJ, et al. Guideline panels should seldom make good practice statements: guidance from the GRADE Working Group. J Clin Epidemiol 2016;80:3-7.

95. Honouring the truth, reconciling for the future: summary of the final report of the Truth and Reconciliation Commission of Canada. Ottawa: Truth and Reconciliation Commission of Canada; 2015. Accessible ici : https://publications.gc.ca/collections/collection_2015/trc/IR4-7-2015-eng. pdf (consulté le 12 nov. 2021).

96. Special Rapporteur on the rights of Indigenous peoples. Geneva: Office of the United Nations High Commissioner for Human Rights (OHCHR). Accessible ici : https://www. ohchr.org/en/issues/ipeoples/srindigenouspeoples/pages/sripeoplesindex.aspx (consulté le 3 mars 2021).

97. Reclaiming power and place: the final report of the national inquiry into missing and murdered Indigenous women and girls. National Inquiry to Missing and Murdered Indigenous Women and Girls; 2019. Accessible ici : https://www. mmiwg-ffada.ca/final-report/ (consulté le 3 mars 2021).

98. Report of the Working Group of Experts on People of African Descent on its mission to Canada. United Nations General Assembly; 2017:1-20.

99. Lewis S. Report of the advisor on race relations to the Premier of Ontario, Bob Rae. Mississauga (ON): Special Investigations Unit; 1992. Accessible ici : https:// www.siu.on.ca/pdfs/report_of_the_advisor_on_race_relations_to_the_premier_ of_ontario_bob_rae.pdf (consulté le 12 nov. 2021).

100. Justice for Joyce: public reports and evidence briefs. Toronto: Well Living House; 2020. Accessible ici : www.welllivinghouse.com/justice-for-joyce-public -reports-and-evidence-briefs/ (consulté le 28 sept. 2021).

101. Williamson EJ, Walker AJ, Bhaskaran K, et al. Factors associated with COVID19-related death using OpenSAFELY. Nature 2020;584:430-6.

102. Brouwers MC, Kho ME, Browman GP, et al.; AGREE Next Steps Consortium. AGREE II: advancing guideline development, reporting and evaluation in health care. CMAJ 2010;182:E839-42.

103. Key health inequalities in Canada: a national portrait - executive summary. Ottawa: Public Health Agency of Canada; modified 2018 Nov. 14. Accessible ici : https:// www.canada.ca/en/public-health/services/publications/science-research-data/ key-health-inequalities-canada-national-portrait-executive-summary.html (consulté le 10 août 2021).

104. Social determinants of health. Geneva: World Health Organization. Accessible ici : https://www.who.int/westernpacific/health-topics/social-determinantsof-health (consulté le 10 août 2021).

105. Moberg J, Oxman AD, Rosenbaum S, et al.; GRADE Working Group. The GRADE Evidence to Decision (EtD) framework for health system and public health decisions. Health Res Policy Syst 2018;16:45.

106. Schünemann HJ, Al-Ansary LA, Forland F, et al.; Board of Trustees of the Guidelines International Network. Guidelines International Network: principles for disclosure of interests and management of conflicts in guidelines. Ann Intern Med 2015;163:548-53.

107. Traversy G, Barnieh L, Akl EA, et al. Managing conflicts of interest in the development of health guidelines. CMAJ 2021;193:E49-54.

108. Canada's First Poverty Reduction Strategy. Employment and Social Development Canada; modified 2021 May 20. Accessible ici : https://www.canada.ca/en/ employment-social-development/programs/poverty-reduction/reports/strategy. html (consulté le 10 août 2021).

109. Marmot M, Allen J, Goldblatt P, et al. Build Back Fairer: the COVID-19 Marmot review - the pandemic, socioeconomic and health inequalities in England. London (UK): Institute of Health Equity; 2020.

110. Turnbull J, Baral S, Bond A, et al. Seeking shelter: homelessness and COVID-19. Ottawa: Royal Society of Canada; 2021.

111. Pottie K, Kendall CE, Aubry T, et al. Clinical guideline for homeless and vulnerably housed people, and people with lived homelessness experience. CMAJ 2020;192:E240-54. 
Intérêts concurrents : Nav Persaud signale être membre du Groupe d'étude canadien sur les soins de santé préventifs, avoir reçu un remboursement de ses frais de déplacement de la part de l'Agence de la santé publique du Canada [ASPC]) et être consultant bénévole auprès du Groupe consultatif pancanadien sur un cadre en vue d'une liste de médicaments. Le $D^{r}$ Persaud déclare aussi avoir reçu des subventions des Instituts de recherche en santé du Canada (IRSC), du Programme des chaires de recherche du Canada et de l'Ontario Strategy for PatientOriented Research (SPOR) SUPPORT Unit. James Dunn signale avoir reçu des honoraires personnels de la Ville de Toronto (Santé publique de Toronto) pour une recherche sur les options en matière de politiques pour des logements salubres. Stephen Hwang signale faire partie à titre bénévole (sans rémunération) du conseil d'administration de Good Shepherd Ministries, être président du comité de surveillance de la sécurité des données pour l'étude Inspire Quitting (financée par le National Cancer Institute, les National Institutes of Health [à titre bénévole, sans rémunération]) et être membre bénévole du Comité de la recherche pour le National Health Care for the Homeless Council (sans rémunération). Le $D^{r}$ Hwang déclare aussi avoir reçu des honoraires à titre d'investigateur principal (IP) du Programme de subvention Fondation des IRSC pour la recherche sur des interventions concernant, l'itinérance, le logement et la santé et en tant qu'IP d'une étude de cohorte sur la COVID-19 et l'itinérance à Toronto soutenue par une subvention de fonctionnement des IRSC. Le Dr Hwang a reçu des honoraires à titre de professionnel de la santé de la part d'Inner City Health Associates (subventionné par le ministère de la Santé et des soins de longue durée de l'Ontario). Le $\mathrm{D}^{r}$ Jonathon Maguire signale avoir reçu des subventions des Instituts de recherche en santé du Canada (IRSC) et de l'Ontario Strategy for Patient-Oriented Research (SPOR) SUPPORT Unit. Andrew Pinto signale avoir fait partie du comité consultatif de l'Alliance pour des communautés en santé, et avoir été consultant bénévole auprès de Muto Health Solutions. Le $D^{r}$ Pinto signale aussi avoir reçu des subventions des IRSC, du gouvernement de l'Ontario, notamment du ministère de la Santé et des Soins de longue durée, de la PSIM Foundation, de la Maytree Foundation, de l'Atkinson Foundation; de la Metcalf Foundation, de la Fondation de l'Hôpital St. Michael et de I'Ontario SPOR SUPPORT Unit. Le Dr Pinto a reçu des honoraires pour des présentations au Joint Centre for Bioethics de l'Université de Toronto (2019), de l'Université McMaster (2020) et de l'Université de Gand (2020). Le Dr Pinto déclare avoir reçu un salaire du Département de médecine familiale et communautaire de l'Hôpital St. Michael, du Département de médecine familiale et communautaire de la Faculté de médecine de l'Université de Toronto et de l'Institut du savoir Li Ka Shing de l'Hôpital St. Michael. Le Dr Pinto a été lauréat de la bourse PSI Graham Farquharson Knowledge Translation Fellowhip en 2019. Patricia O'Campo signale faire partie du conseil d'administration de Centraide du Grand Toronto, qui subventionne des agences qui desservent les femmes victimes de violence conjugale. $\mathrm{M}^{\mathrm{me}} \mathrm{O}^{\prime} \mathrm{Campo}$ signale aussi avoir reçu deux subventions des IRSC comme détentrice de la Chaire de recherche du Canada sur les approches interventionnelles dans le domaine de la santé des populations et du Conseil de recherches en sciences humaines pour des projets sur la violence conjugale. Sean Rourke signale avoir reçu des honoraires de consultation en tant qu'expert de la mise en œuvre stratégique pour la Fondation canadienne de recherche sur le sida. M. Rourke déclare également des subventions des IRSC et de l'ASPC, versées à l'Hôpital St. Michael, et une subvention de l'Annual Foundation, versée à la Fondation canadienne de recherche sur le sida (et non à M. Rourke). Daniel Werb signale avoir reçu une bourse salariale de nouveau chercheur des IRSC et une subvention de la Fondation de l'Hôpital St. Michael. M. Werb est aussi en attente d'un brevet pour un dispositif de vérification des médicaments. Aucun autre intérêt concurrent n'a été déclaré.

Cet article a été révisé par des pairs.

Affiliations : Centre MAP pour des solutions en santé urbaine (Persaud, Wood, Workentin, Adekoya, Dunn, Hwang, Maguire, Pinto, O'Campo, Rourke, Werb), Hôpital St. Michael du Réseau hospitalier Unity Health Toronto; Faculté de médecine (Persaud, Hwang, Maguire, Pinto) et Département de psychiatrie (Rourke), Faculté de médecine, Université de Toronto, Toronto, Ont.

Collaborateurs : Tous les auteurs sont membres du Comité des recommandations chargé de la préparation de la ligne directrice. Nav Persaud a contribué à la conception et à la modélisation du travail et à l'interprétation des données; il a rédigé le manuscrit et en a révisé de façon critique le contenu intellectuel important. Hannah Woods et Aine Workentin ont contribué à l'acquisition et à l'interprétation des données, rédigé le manuscrit et en ont révisé de façon critique le contenu intellectuel important. Itunu Adekoya a contribué à l'acquisition et à l'interprétation des données. James Dunn, Stephen Hwang, Jonathon Maguire, Andrew Pinto, Patricia O'Campo, Sean Rourke et Daniel Werb ont contribué à la modélisation, à l'interprétation, à la rédaction de l'ébauche du manuscrit et à sa révision de façon critique pour tout contenu intellectuel important. Tous les auteurs ont donné leur approbation finale pour la version soumise pour publication et assument l'entière responsabilité de tous les aspects du travail.

Financement : La préparation de cette ligne directrice a bénéficié du soutien de la Fondation de l'Hôpital St. Michael qui n'a participé ni à la conception, ni au développement, ni à la diffusion des recommandations.

Appuis : Cette ligne directrice est approuvé par Black Physicians of Canada, Médecins canadiens pour le régime public et l'Association médicale canadienne. Le Collège des médecins de famille du Canada appuie ces recommandations.

Propriété intellectuelle du contenu : Il s'agit d'un article en libre accès distribué conformément aux modalités de la licence Creative Commons Attribution (CC BY-NC-ND 4.0), qui permet l'utilisation, la diffusion et la reproduction dans tout médium à la condition que la publication originale soit adéquatement citée, que l'utilisation se fasse à des fins non commerciales (c.-à-d., recherche ou éducation) et qu'aucune modification ni adaptation n'y soit apportée. Voir : https://creativecommons. org/licenses/by-nc-nd/4.0/deed.fr.

Partage des données : Toutes les données sont accessibles dans l'article et ses annexes.

Remerciements : Les auteurs remercient Janet Brown, Pamela McDonald-Kuhne et Suzanne Zerger pour leur soutien à la préparation de cette ligne directrice, y compris l'implication des parties prenantes. Ils remercient également Carolyn Zeigler pour son aide pour l'interrogation de la documentation.

Déclaration d'intérêts : Nav Persaud est corédacteur pour le CMAJ, mais n'a pas participé au processus ayant mené au choix de cet article.

Correspondance : Nav Persaud, nav.persaud@utoronto.ca 